\title{
Silver-based reflective coatings for micromachined optical filters
}

\section{Bartek $\dagger$, J H Correia and R F Wolffenbuttel}

Delft University of Technology, Faculty for Information Technology and Systems, Laboratory for Electronic Instrumentation/DIMES, Mekelweg 4, 2628 CD Delft, The Netherlands

Received 12 February 1999

Abstract. Silver films of $40 \mathrm{~nm}$ thickness, evaporated on a $300 \mathrm{~nm}$ thick low-stress silicon nitride layer, are used as high-quality mirrors operating in the visible and near IR spectral range. Application of a silicon nitride membrane under tension, placed within a square $\mathrm{Si}$ frame after bulk micromachining, improves the initial mirror flatness. Two parallel mirrors, each with square aperture of up to $2 \times 2 \mathrm{~mm}^{2}$ and an electrostatically controlled spacing, form a tunable Fabry-Pérot optical filter. Investigation of the silver-based reflective coatings, and mirror characterization, including influence of bulk micromachining, are presented.

\section{Introduction}

In recent years much research has been directed towards the development of tunable micromachined optical filters and based on a Fabry-Pérot (F-P) resonator [1-3]. Research has been primarily focused on the near-infrared region (wavelength of 1.3 and $1.55 \mu \mathrm{m}$ ), because of interest in multi-mode optical fiber communication. In this application operation in a narrow optical band and the transparency of the Si substrate in the spectral region of interest facilitate the system design.

Attempts to fabricate a device in the visible spectral range have also been reported [4], but were less successful. The goal is to develop an integrated spectrometer. In such an application, the requirements are much more demanding. Wide optical band operation is required (preferably over the entire visible spectral range $400-800 \mathrm{~nm}$ ). Furthermore, the mirror flatness becomes more critical due to the decreased wavelength.

The most important part of the F-P device is the mirrors. Two types of reflective coating are used: dielectric and metallic. The dielectric mirrors, if properly designed and fabricated, feature high performance (high reflectivity, low absorption losses), but their nature (distributed character) requires the deposition of a sequence of two (or more) different dielectric materials with well controlled thickness, forming a stack of many layers. To be effective in a wide optical band, usually more than 15 layers for one mirror are required [5]. This complicates fabrication.

Although the metallic-based coatings have much higher losses, these can be attractive in certain applications due to simplicity of fabrication (only one layer must be deposited). Another advantage is that metallic mirrors generally perform well over a wide spectral range. Aluminum, gold and silver are the most used metals for reflective coatings. Figure 1

$\dagger$ Phone: +31-15-2786287. Fax: +31-15-2785755.

E-mail address: M.Bartek@its.tudelft.nl.

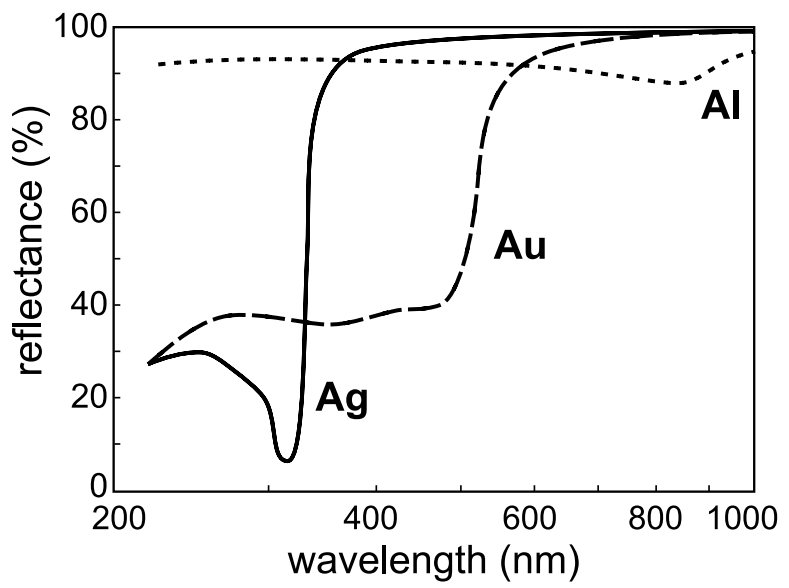

Figure 1. Reflectance of silver, gold and aluminum as a function of the radiation wavelength, after [5].

shows their reflectance in the near-UV, visible and near-IR spectral region [5].

Al would be the most suitable material in terms of fabrication compatibility, but unfortunately Al has higher absorption losses than $\mathrm{Ag}$ or $\mathrm{Au}$ in the visible and nearIR. For the visible and near-IR spectral regions, silver is the best choice, but exhibits poor long-term stability (tendency to tarnishing). Gold is more corrosion resistant than silver and would be the best choice if it were not for its poor visible and ultraviolet performance. Unlike macroscopic applications of silver-based reflective coatings [6], the poor environmental resistance of silver is not critical in a microsystem application. Sealing of a complete system would avoid any environmentally caused mirror degradation. Another advantage is that Ag is a natural low-pass filter, cutting off the UV range (see figure 1). Silver-based mirrors have been previously reported for use in non-tunable distributed filters composed of a wedge shaped dielectric film 


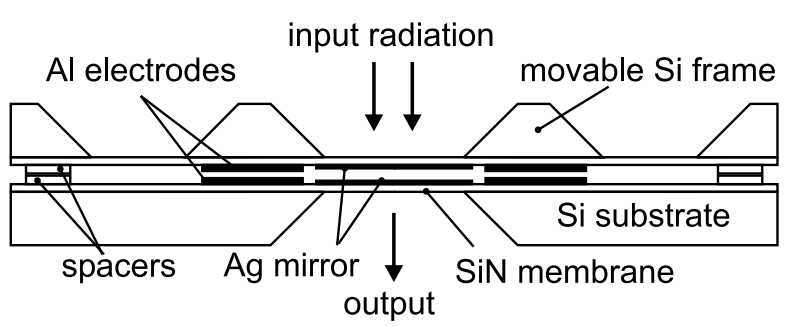

Figure 2. Cross-section of the proposed micro-machined Fabry-Pérot optical filter.

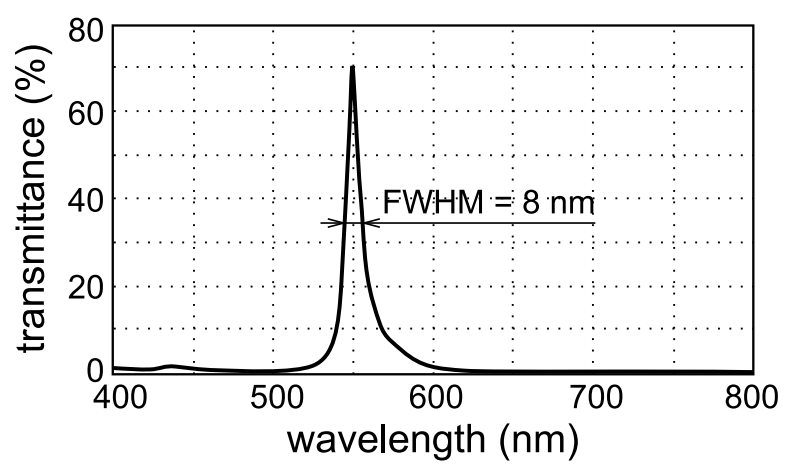

Figure 3. Calculated transmittance for $300 \mathrm{~nm} \mathrm{SiN}-40 \mathrm{~nm}$ Ag-500 nm air-40 nm Ag-300 nm SiN layer stack.

sandwiched between two reflective (Ag) thin films [7].

This paper presents results on the investigation of the feasibility of silver-based reflective coatings, with the goal to develop a miniature $\mathrm{F}-\mathrm{P}$ based spectrometer for use in the visible spectral range.

\section{Micromachined Fabry-Pérot optical filter}

For initial tests a device similar to that of Raley et al [4] was chosen. Fabrication is based on bulk micromachining as shown schematically in cross-section in figure 2. The tunable Fabry-Pérot optical filter is formed by two parallel $40 \mathrm{~nm}$ thick silver mirrors supported by a $300 \mathrm{~nm}$ low-stress silicon nitride membrane with a square aperture of $2 \times 2 \mathrm{~mm}^{2}$ and an initial cavity gap of $1.2 \mu \mathrm{m}$. One of the mirrors is fixed; the other is under tension on a movable $\mathrm{Si}$ frame, which is electrostatically deflected using several distributed electrodes to control the mirror spacing and parallelism.

A thin film optics software package (TFCalc 3.2.5) was used to perform optimization of the mirror layer thickness, composition and order (optical data in [8]). The silver layers must be inside the resonance cavity to avoid excessive absorption losses in the silicon nitride layer during multiple reflections. The simulated transmittance for the optimized $40 \mathrm{~nm} \mathrm{Ag/300} \mathrm{nm} \mathrm{SiN} \mathrm{mirrors} \mathrm{with} \mathrm{a} 500 \mathrm{~nm}$ spacing (air gap cavity) is shown in figure 3. The Ag layer thickness is a trade-off between achievable FWHM (full width at half maximum) and absorption loss. This is illustrated in figure 4, where simulated transmittance and finesse as a function of Ag-layer thickness are shown.

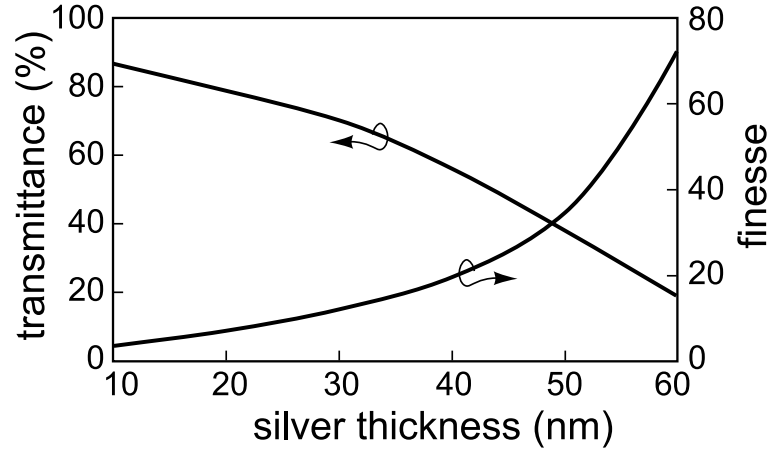

Figure 4. Simulated transmittance and finesse as a function of Ag-layer thickness.

\section{Fabrication}

On the same wafer (100 mm, double-side polished), upper and bottom dies have been fabricated using a five-mask process (see figure 5). Firstly, $400 \mathrm{~nm}$ recesses are formed using LOCOS. Subsequently, a $300 \mathrm{~nm}$ low-stress $(<0.15 \mathrm{GPa})$ LPCVD silicon nitride layer is deposited and protected by a $300 \mathrm{~nm}$ LPCVD poly-Si layer. Then, PECVD oxide $(0.3-1 \mu \mathrm{m})$ is deposited on a wafer front-side with thickness corresponding to the required initial resonance cavity gap. The PECVD oxide/poly-Si stack is patterned to form spacers between upper and bottom dies for later die attachment. The $300 \mathrm{~nm} \mathrm{Al} \mathrm{interconnect} \mathrm{and} \mathrm{control/sensing}$ electrodes (deposited by sputtering) are 'buried' in $400 \mathrm{~nm}$ recesses to increase the initial spacing of the electrodes and avoid sticking during operation. The wafer back-side is patterned to prepare windows for anisotropic $\mathrm{KOH}$ etching. Silver mirror layers are e-beam evaporated and patterned using lift-off on the wafer front-side. The anisotropic $\mathrm{KOH}$ etching ( $33 \mathrm{wt} \% \mathrm{KOH}$ solution at $85^{\circ} \mathrm{C}$ ) is performed in a holder to protect the Ag mirrors. To facilitate wafer dicing into the individual dies, deep $\mathrm{V}$-shaped trenches are formed during $\mathrm{KOH}$ etching. After the bottom die is mounted on a $\mathrm{PCB}$, the upper die is attached and fixed using glue.

\section{Results}

To test the concept of silver-based mirrors, a $40 \mathrm{~nm} \mathrm{Ag} \mathrm{layer}$ was deposited on $\mathrm{SiN}$ membranes from one or both sides after bulk micromachining. The interference measurements (projected interference fringes) showed an excellent initial mirror flatness. The LPCVD silicon nitride membrane, which is under tension after the release using anisotropic $\mathrm{KOH}$ etching, improves the initial flatness of the silver mirror.

The optical response was measured using a large area photodiode $\left(5.1 \mathrm{~mm}^{2}\right)$ and HP4142B DC source/monitor controlled by an HP 9000/700 computer. A $100 \mathrm{~W}$ tungsten lamp and Oriel 77250 monochromator with a ruled grating were used as a light source.

Figure 6 shows the simulated and measured transmittance for a $40 \mathrm{~nm} \mathrm{Ag/450} \mathrm{nm} \mathrm{SiN/40} \mathrm{nm} \mathrm{Ag} \mathrm{layer} \mathrm{stack.}$ An SEM photograph of the surface morphology of an on$\mathrm{SiN}$ evaporated silver layer is shown in figure 7. From AFM measurements (figure 8) a typical RMS roughness of about $15 \mathrm{~nm}$ was determined. 

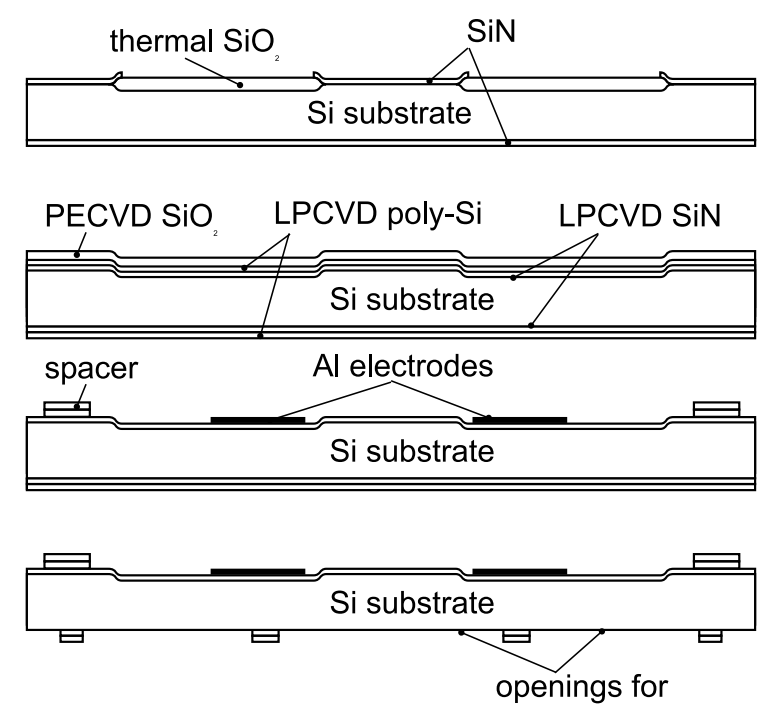

Ag mirror anisotropic $\mathrm{KOH}$ etching
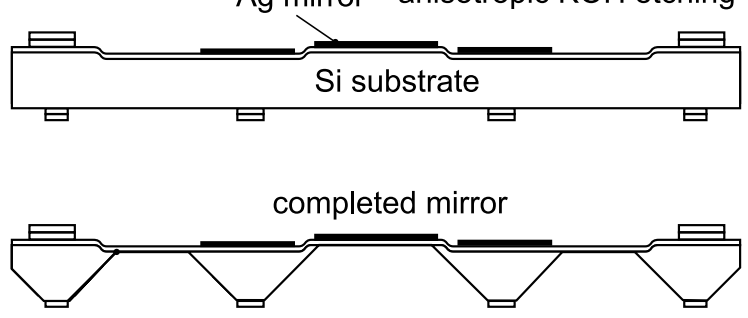

Figure 5. Schematic fabrication sequence (see text for details).

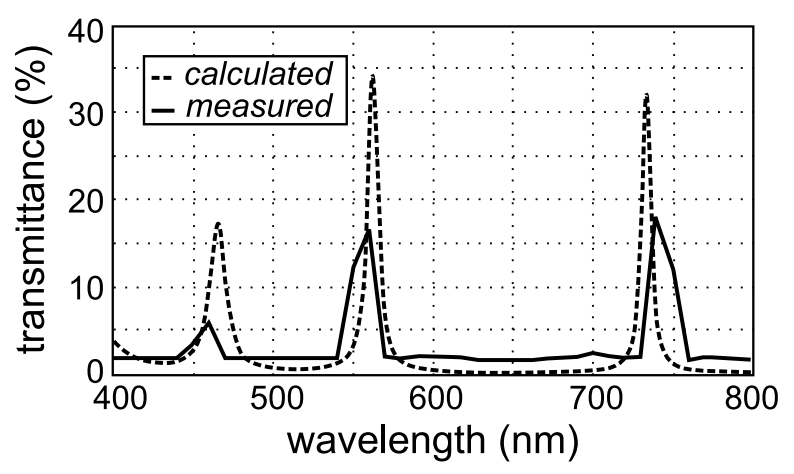

Figure 6. Calculated and measured transmittance for $40 \mathrm{~nm}$ $\mathrm{Ag}-450 \mathrm{~nm} \mathrm{SiN}-40 \mathrm{~nm}$ Ag layer stack.

The initial complete fabrication tests resulted in stained mirrors. This was found to be due to problems with cleaning after anisotropic $\mathrm{KOH}$ etching. In the fabrication sequence the Ag mirrors were patterned using lift-off in an ultrasonic bath using acetone. Three cascaded baths with fresh clean acetone yield clean Ag mirrors. For protection purposes the wafer was subsequently covered by a photoresist layer to avoid any mechanical damage to the wafer surface during further processing.

The anisotropic $\mathrm{KOH}$ etching was performed using a sealed stainless steel holder. After the SiN membranes were completely cleared from $\mathrm{Si}$, the wafer in the holder was carefully taken out from the $\mathrm{KOH}$ solution and rinsed for $30 \mathrm{~min}$ in de-ionized water. The sealed holder was then opened and the wafer was separately rinsed again for $30 \mathrm{~min}$. The protection photoresist layer on the wafer front side was

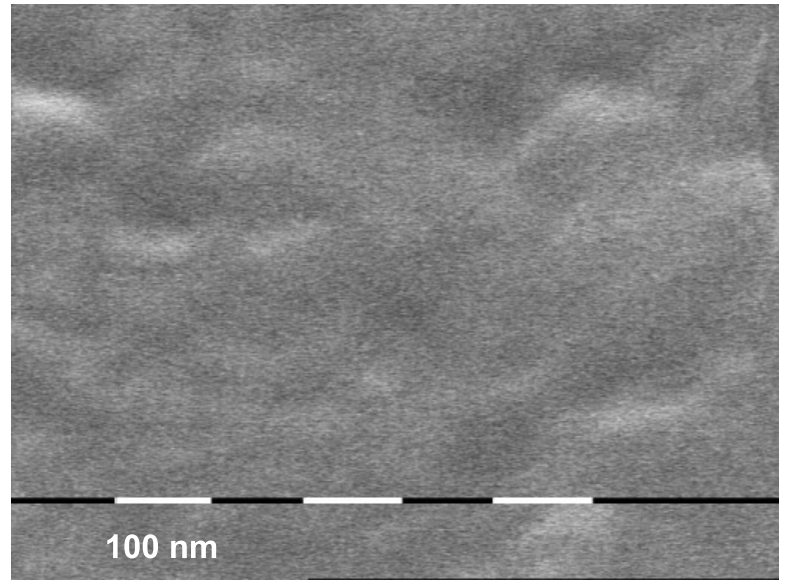

Figure 7. SEM photograph of the evaporated silver-layer surface.

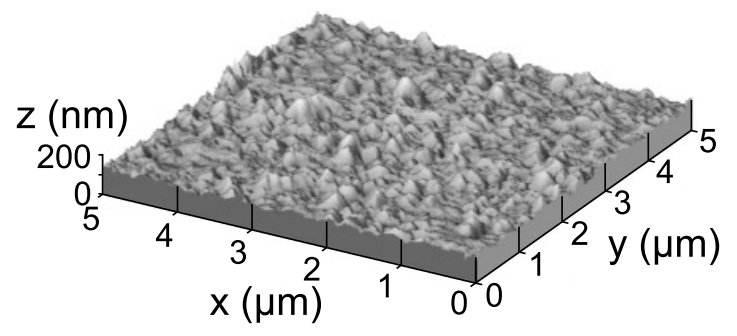

Figure 8. AFM surface scan of a $40 \mathrm{~nm}$ silver layer evaporated on a $300 \mathrm{~nm}$ silicon nitride membrane.

subsequently removed in acetone. The resulting structures (Si frame hanging on a $\mathrm{SiN}$ membrane) are very fragile and extreme care must be taken during further handling. Therefore, any 'aggressive' drying method is not allowed. Slow drying in a nitrogen flow resulted in stains on most of the membranes. These were found to be probably organic residue from the protective photoresist layer. Improvement was achieved using isopropyl alcohol after acetone cleaning, or by completely avoiding the protective photoresist layer. However, the best solution will be deposition of the Ag layer at the very end of the fabrication sequence. This improvement is incorporated into the fabrication sequence for our next generation of the F-P devices. Figure 9 shows a photograph of the fabricated miniature F-P device. The square-shaped pyramidal openings at the periphery of the device are used during the assembly of the bottom and upper die to perform the manual alignment.

On all assembled devices, a small number of interference fringes in the transmitted light could be observed. This indicates an initial non- parallelism of the mirrors. Figure 10 shows the measured reflectance of an assembled device without adjusting the mirror parallelism. This measurement was performed using a microscope-based spectroscopic system in a focused light spot with a diameter of about $100 \mu \mathrm{m}$. Without the light beam focusing, no resonance peaks could be observed.

To achieve any resonance peaks for an unfocused light source, the mirrors must be perfectly parallel. A manual adjustment of the mirror parallelism, as used in this study, is a rather challenging task. One possible way is to use a small laser pointer and to project the multiple reflections 


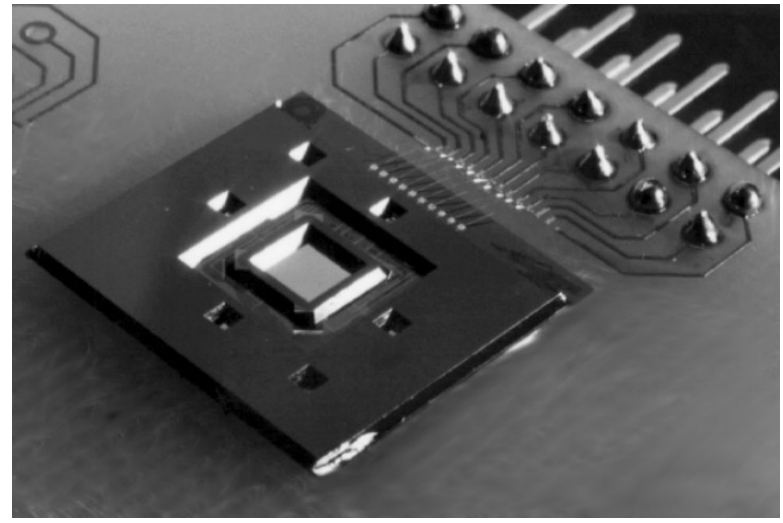

Figure 9. A photograph of the fabricated miniature Fabry-Pérot tunable interferometer device.

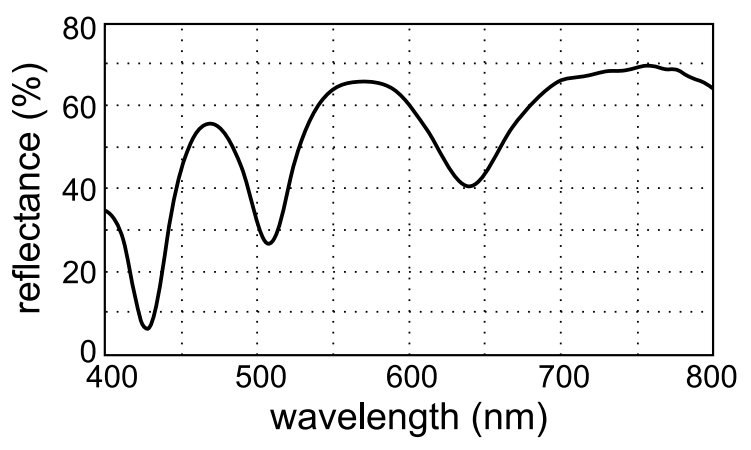

Figure 10. Reflectance of F-P filter measured directly after assembly without adjustment of mirror parallelism. The measurement was performed using a focused light spot $(\sim 100 \mu \mathrm{m}$ in diameter).

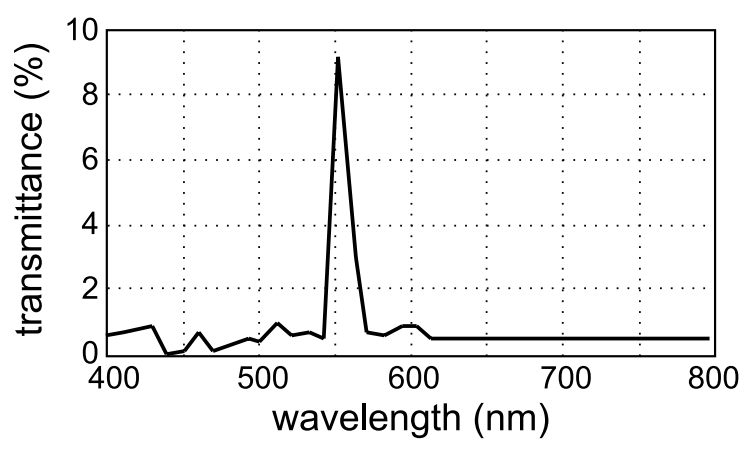

Figure 11. The measured transmittance for an air cavity gap of $500 \mathrm{~nm}$ and silver mirrors with thickness of $40 \mathrm{~nm}$ after the mirror parallelism was manually adjusted.

on a wall. Then by adjusting the voltages on the control electrodes, mirror parallelism can be achieved. Simultaneous parallelism adjustment and tuning of the cavity spacing is feasible only when a computerized system for the voltage control is used.

The measured transmittance for an air cavity gap of about $500 \mathrm{~nm}$ is shown in figure 11. When compared to the simulation (see figure 3 ), the measured transmittance is reduced by a factor of 8 . The most probable explanation is the imperfection of the $\mathrm{F}-\mathrm{P}$ resonator structure. Light scattering (originated by the mirror roughness) will result in a decreased resonator quality with a decreased resonance peak. Also, an increased mirror thickness will cause peak height reduction due to a higher absorption. Nevertheless, a transmittance of about $10 \%$ and an FWHM less than $20 \mathrm{~nm}$ was feasible.

\section{Conclusions}

Silver films of $40 \mathrm{~nm}$ thickness, evaporated on a $300 \mathrm{~nm}$ thick low-stress silicon nitride layer (which is, after anisotropic etching in $\mathrm{KOH}$, under tension on a square silicon frame), have been investigated for use as a high-quality mirrors operating in the visible and near-IR spectral range. Two parallel mirrors, with square aperture of up to $2 \times 2 \mathrm{~mm}^{2}$ and an electrostatically controlled spacing, form a tunable Fabry-Pérot optical filter. Special care is required to maintain high mirror quality, if a silver layer is to be deposited before anisotropic $\mathrm{KOH}$ etching. The best choice is to deposit silver at the very end of the fabrication sequence. For our present device that is not possible and care must be taken to protect the silver layer from degradation during the anisotropic $\mathrm{KOH}$ etching and subsequent cleaning. Optical measurements show that, for perfectly parallel mirrors, transmittance of about $10 \%$ and an FWHM of less than $20 \mathrm{~nm}$ was achieved. The adjustment of the mirror parallelism, however, is not a trivial task.

\section{Acknowledgments}

The authors wish to thank the staff of Delft Institute of Microelectronics and Submicron Technology (DIMES), especially $\mathrm{J}$ Groeneweg, for technical assistance in fabrication of the devices. The work presented here has been supported in part by the Dutch Technology Foundation (STW), project No DEL 55.3733.

\section{References}

[1] Tran A T T D, Lo Y H, Zhu Z H, Haronian D and Mozdy E 1996 Surface micromachined Fabry-Pérot tunable filter IEEE Photon. Technol. Lett. 8 393-5

[2] Christenson G L, Tran A T T, Zhu Z H and Lo Y H 1997 Surface micromachined long wavelength LED/photodetector with a continuous tuning range of 75 nm Proc. MEMS'97 (Nagoya, 1997) pp 61-5

[3] Patterson J and van Zeghbroeck B 1996 Fabrication and analysis of $\mathrm{Si} / \mathrm{SiO}_{2}$ micro-mechanical modulators Digest IEEE/LEOS 1996 Summer Topical Meeting on Optical MEMS and their Applications (Monterey, CA, 1996) pp 25-6

[4] Raley N F, Ciarlo D R, Koo J C, Beiriger B, Trujillo J, Yu C, Lomis G and Chow R 1992 A Fabry-Pérot microinterferometer for visible wavelength Proc. 5th IEEE Solid State Sensor and Actuator Workshop (Hilton Head Island, SC, 1992) pp 170-3

[5] Macleod H A 1986 Thin-Film Optical Filters 2nd edn (Bristol: Hilger)

[6] Song D-Y, Sprague R W, Macleod H A and Jacobson M 1985 Progress in the development of a durable silver-based high-reflectance coating for astronomical telescopes Appl. Opt. 24 1164-70

[7] Holm-Kennedy J W, Tsang K W, Sze W W, Jiang F and Yang D 1991 A novel monolithic, chip-integrated, color spectrometer: the distributed wavelength filter component Proc. SPIE 1527 322-31

[8] Poenar D P and Wolffenbuttel R F 1997 Optical properties of thin-film silicon-compatible materials Appl. Opt. 36 5122-8 